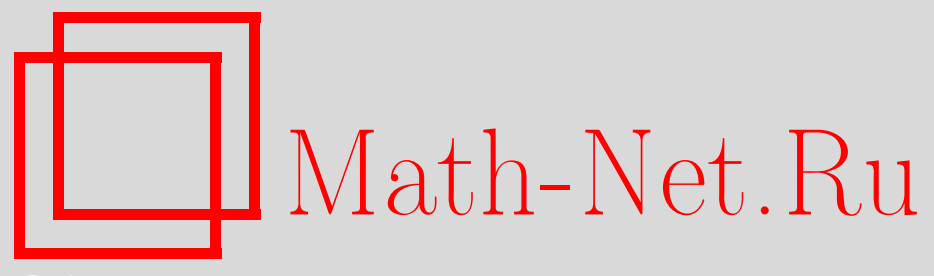

Т. М. Алиашвили, Г. Н. Химшиашвили, Об эйлеровой характеристике пересечения квадрик, УМH, 2006, том 61, выпуск 3, 163-164

DOI: https://doi.org/10.4213/rm1700

Использование Общероссийского математического портала Math-Net.Ru подразумевает, что вы прочитали и согласны с пользовательским соглашением http://www . mathnet.ru/rus/agreement

Параметры загрузки:

IP : 3.93.64.190

26 апреля 2023 г., 13:10:56

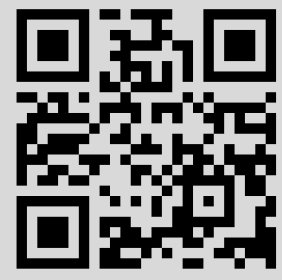




\section{Об эйлеровой характеристике пересечения квадрик}

\section{Т. М. Алиашвили, Г. Н. Химшиашвили}

1. Пусть $\chi(n, m, d)$ обозначает совокупность всевозможных значений эйлеровой характеристики непустых компактных алгебраических множеств в $\mathbb{R}^{n}$, представимых как пересечение $m$ алгебраических гиперповерхностей степени $d$. Под алгебраической гиперповерхностью степени $d$ в $\mathbb{R}^{n}$, как обычно, понимается множество всех вещественных нулей некоторого многочлена степени $d$ от $n$ переменных с вещественными коэффициентами [1].

В ряде вопросов вещественной алгебраической геометрии, связанных с 16-й проблемой Гильберта и неравенствами Петровского-Олейник, возникает задача описания множеств $\chi(n, m, d)$ для различных значений параметров $n, m, d$, которая остается нерешенной как в общем виде, так и для сравнительно небольших значений параметров [1]. Представляет интерес также описание множеств вида $\chi(n, d)\left(\chi_{\pitchfork}(n, d)\right)$, возникающих при рассмотрении пересечений (трансверсальных) гиперповерхностей степени $d$, взятых в произвольном конечном числе [2]-[5]. Каждое из полученных множеств будем называть $\chi$-спектром соответствующего типа.

Мы приводим оценки спектра $\chi(n, 2)$ всевозможных значений эйлеровой характеристики непустого компактного пересечения вещественных квадрик в евклидовом пространстве заданной размерности $n$. Из общих результатов вещественной алгебраической геометрии следует, что множество $\chi(n, 2)$ конечно, и поэтому представляет интерес нахождение как можно более точных верхней и нижней оценок для $\chi(n, 2)$ и $\chi_{\pitchfork}(n, 2)$. Точную (т.е. достижимую) оценку снизу (сверху) будем называть левой (правой) границей. Удобно говорить об оценках границ снаружи и изнутри. Оценки снаружи могут быть выведены из результатов работ [6] и [7], тогда как получение оценок изнутри требует рассмотрения конкретных примеров, что являлось прерогативой первого автора.

2. Приведем вначале общую оценку эйлеровой характеристики, применимую к пересечениям алгебраических гиперповерхностей произвольных степеней. Напомним, что для натуральных $n, k_{1}, \ldots, k_{n}$ число Петровского $P\left(n ; k_{1}, \ldots, k_{n}\right)$ определяется как число всех целых точек в параллелепипеде $B=\prod\left[0, k_{i}-1\right]$, лежащих на гиперплоскости $\left\{2\left(x_{1}+\cdots+x_{n}\right)=k_{1}+\cdots+k_{n}-n\right\}$ [3], [7]. Если все числа $k_{i}$ равны некоторому числу $k$, то соответствующее число Петровского обозначается $P(n ; k)$. Согласно основному результату [7], абсолютная величина топологической степени полиномиального эндоморфизма, компоненты которого имеют алгебраические степени $k_{1}, \ldots, k_{n}$, не превосходит $P\left(n ; k_{1}, \ldots, k_{n}\right)$.

Теорема 1. Удвоенная абсолютная величина эйлеровой характеристики компактного пересечения произвольного числа вещественных гиперповерхностей степени $d$ в $\mathbb{R}^{n}$ не превосходит увеличенного на единичу числа Петровского $P(n+1 ; 2 d+3)$.

Это следует из явной формулы для эйлеровой характеристики, полученной в [6], и вышеупомянутого результата работы [7].

СлеДСтвиЕ 1. Абсолютная величина любого элемента спектра $\chi(n, 2)$ не превосходит половины увеличенного на единицу числа сочетаний из $4 n+3$ nо $n$.

3. Для уточнения структуры $\chi$-спектров можно также использовать результаты [8]-[11]. Рассмотрим собственное квадратичное отображение $Q: \mathbb{R}^{n} \rightarrow \mathbb{R}^{m}$ и обозначим через $\chi(Q)$ множество значений эйлеровой характеристики непустых слоев $Q$. Ясно, что $\chi(n, m, 2)$ является объединением множеств типа $\chi(Q)$. Это обстоятельство полезно, поскольку из результатов [8]-[10] следует, что $\chi(Q)$ может быть эффективно 
найдено по коэффициентам $Q$. Следующие два предложения получаются из результатов [11] и [12].

Теорема 2. Для любого невырожденного квадратичного отображения $Q: \mathbb{R}^{n} \rightarrow$ $\mathbb{R}^{m}$ существуют вещественные многочлены $f_{j}$ от $m$ переменных в четном числе $s$ такие, что спектр $\chi(Q)$ совпадает с множеством значений суммы их знаков по всем точкам $\mathbb{R}^{m}$.

СледствиЕ 2. Обе границы спектра $\chi(n, 2)$ четны.

Очевидно, что $\chi(n, 2) \subseteq \chi(n+1,2)$ и правая граница спектра $\chi(n, 2)$ заведомо не меньше $2^{n}$ (достаточно взять $n$ пар параллельных гиперплоскостей общего положения). Для всех известных нам $\chi$-спектров данная оценка точна.

Легко показать, что спектр $\chi(n, 2)$ всегда содержит целочисленный сегмент $\left[0,2^{n}\right] \cap$ $\mathbb{Z}$. По-видимому, такой спектр всегда совпадает с целочисленным сегментом. Комбинируя приведенные факты и результаты [2], [4], [12], можно оценить и вычислить спектры $\chi(n, 2)$ при малых $n$. Например:

$$
\begin{gathered}
\chi(2,2)=[0,4] \cap \mathbb{Z}, \quad \chi(3,2)=[-2,8] \cap \mathbb{Z}, \quad \chi(4,2) \supseteq[-2,16] \cap \mathbb{Z}, \\
\chi(5,2) \supseteq[-4,32] \cap \mathbb{Z}, \quad \chi(6,2) \supseteq[-6,64] \cap \mathbb{Z} .
\end{gathered}
$$

\section{Список литературы}

[1] L. Blum, F. Cucker, M. Shub, S. Smale, Complexity and real computation, Springer-Verlag, New York, 1998. [2] А. А. Аграчёв, Р. В. Гамкрелидзе, Итоги науки. Соврем. проблемы матем. Новейшие достижения, 35, 1989, 179-239. [3] В.И. Арнольд, Функи. анализ и его прил., 12:1 (1978), 1-14. [4] G. N. Khimshiashvili, Proc. A. Razmadze Math. Inst., 125 (2001), 1-121. [5] G. N. Khimshiashvili, Georgian Math. J., 11:4 (2004), 759-770. [6] J. Bruce, Bull. London Math. Soc., 22:6 (1990), 547-552. [7] А.Г. Хованский, Функц. анализ и его прил., 13:1 (1979), 49-58. [8] Г. Н. Химшиашвили, Сообщ. АН ГССР, 85:2 (1977), 309-312. [9] D. Eisenbud, H. I. Levine, Ann. of Math. (2), 106:1 (1977), 19-44. [10] T. Aliashvili, J. Math. Sci. (New York), 118:5 (2003), 5325-5346. [11] A. Parusiński, Z. Szafraniec, Banach Center Publ., 44 (1998), 175-182. [12] Y. Kamiyama, Kyushu J. Math., 54:2 (2000), 333-369.

Т. М. Алиашвили (Т. M. Aliashvili)

Математический институт им. А. М. Размадзе АН Грузии

Г. Н. Химшиашвили (G. N. Khimshiashvili)

Математический институт им. А. М. Размадзе АН Грузии

E-mail: gogikhim@yahoo.com, khimsh@rmi.acnet.ge
Представлено С. М. Гусейн-Заде Принято редколлегией 01.12 .2005 